\title{
Some hypersonic intake studies
}

\author{
F. Lanson and J. L. Stollery \\ College of Aeronautics \\ Cranfield University \\ Bedford, UK
}

\section{ABSTRACT}

A 'two dimensional' air intake comprising a wedge followed by an isentropic compression has been tested in the Cranfield Gun Tunnel at Mach $8 \cdot 2$. These tests were performed to investigate qualitatively the intake flow starting process. The effects of cowl position, Reynolds number, boundary-layer trip and introduction of a small restriction in the intake duct were investigated. Schlieren pictures of the flow on the compression surface and around the intake entrance were taken.Results showed that the intake would operate over the Reynolds number range tested.

Tests with a laminar boundary layer demonstrated the principal influence of the Reynolds number on the boundary-layer growth and consequently on the flow structure in the intake entrance. In contrast boundary layer tripping produced little variation in flow pattern over the Reynolds number range tested. The cowl lip position appeared to have a strong effect on the intake performance. The only parameter which prevented the intake from starting was the introduction of a restriction in the intake duct.

The experimental data obtained were in good qualitative agreement with the CFD predictions. Finally, these experimental results indicated a good intake flow starting process over multiple changes of parameters.
\end{abstract}

\section{NOMENCLATURE}

C constant in the linear temperature-viscosity relation

$L \quad$ length of the separated region on the cowl internal surface ( $\mathrm{mm}$ )

M Mach number

p static pressure $\left(\mathrm{N} / \mathrm{m}^{2}\right)$

$\mathrm{Re}$. Reynolds number

$T$ temperature $(\mathrm{K})$

$u \quad$ velocity $\left(\mathrm{ms}^{-1}\right)$

$x \quad$ axial distance from the ramp leading edge ( $\mathrm{mm})$ vertical distance of the cowl lip from the top of the step ( $\mathrm{mm}$ )

$\delta \quad$ boundary layer thickness ( $\mathrm{mm})$ and flow deflection angle (deg)

$\delta$ * boundary layer displacement thickness $(\mathrm{mm})$

$\theta \quad$ shock wave angle (deg)

$\mu \quad$ viscosity $\left(\mathrm{Ns} / \mathrm{m}^{2}\right)$

Mach angle (deg)

$\rho$

density $\left(\mathrm{kg} / \mathrm{m}^{3}\right)$

\section{Subscripts}

total conditions generated at entry to the convergent divergent nozzle 

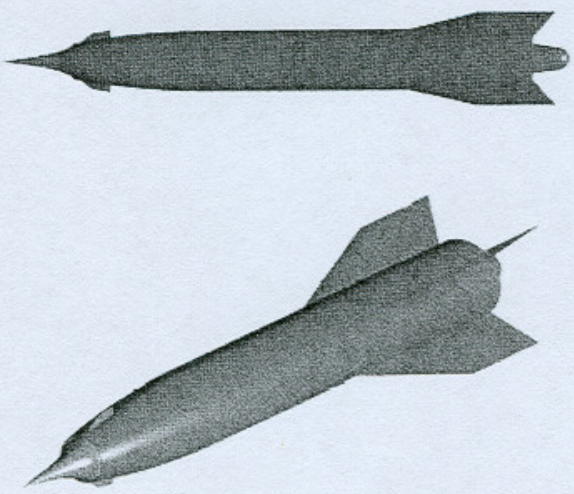

Figure 1. Artist's impression of SHyFE from Ref.1.

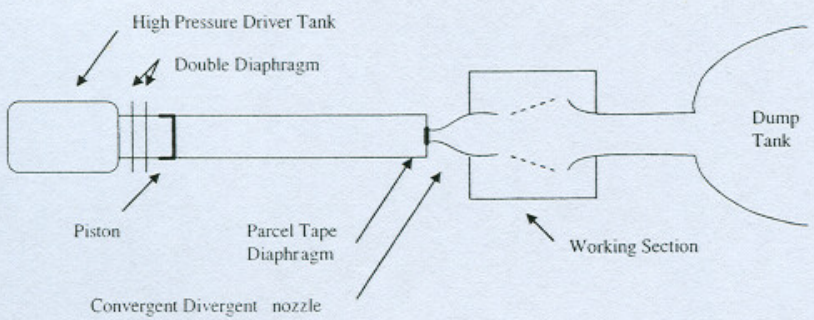

Figure 2. Layout of the gun tunnel. freestream conditions, i.e. conditions at the nozzle exit conditions behind the wedge oblique shock driver conditions

wall surface conditions

referenced to $x$-distance

\subsection{INTRODUCTION}

Hypersonic vehicles are so tightly integrated that the conventional aerospace practice of independent component development is unlikely to result in a viable aircraft. The USA recognised the need to build a small scale test vehicle to establish design methods following the collapse of the X-30 project (The National Aerospace Plane, NASP). NASA then built the smaller X-43 which has recently flown successfully at Mach 7 and 10 powered by a ten second burn from a hydrogen fuelled scramjet.

The UK programme is SHyFE, the sustained hypersonic flight experiment where the emphasis is on the word 'sustained'. The vehicle mass, and the project budget, is more than an order of magnitude less than for the X-43 but the most significant difference is that SHyFE is designed to achieve a steady state hypersonic cruise at Mach 6 with the vehicle temperatures in equilibrium during the flight time of about three minutes. A ramjet with subsonic combustion of kerosene fuel will be used to reduce the engine development risk. An early configuration is shown in Fig. 1. The aircraft is rocket boosted to Mach 4 at a height of $15 \mathrm{~km}$ before the ramjet is ignited. SHyFE wil then accelerate and climb to an altitude of $32 \mathrm{~km}$. where it will cruise at Mach 6 for about $300 \mathrm{~km}$. before the fuel is spent.

\subsection{EXPERIMENTAL APPARATUS}

The tests were made using the Cranfield gun ttunnel. This facility is an intermittent, free piston compression heater feeding a blowdown hypersonic tunnel, (Fig. 2).

The light aluminium alloy piston is driven by compressed air at drive pressures $\left(P_{D}\right)$ up to 137 atmospheres $(2,000 \mathrm{psig})$ contained in a $0.3 \mathrm{~m}^{3}$ (4cu.ft) high-pressure-vessel. The compression tube is a $6.1 \mathrm{~m}$ (20ft.) long, $8 \mathrm{~cm}(3 \cdot 2 \mathrm{inch})$ bore barrel, giving an initial test gas volume of about $0.03 \mathrm{~m}^{3}\left(1 \mathrm{ft}^{3}\right)$. The drive vessel is coupled to the barrel via a double diaphragm rig. Primary diaphragms are of unscribed commercial grade aluminium sheet, while 'parcel tape' is used for the secondary diaphragm at the nozzle end of the barrel. Two contoured nozzles are available providing uniform flow at either $\mathrm{M}=8 \cdot 2$ or $\mathrm{M}=12 \cdot 2$. Both nozzles have an exit diameter of $20 \mathrm{~cm}$. ( 8 inches) giving a useful core of about $15 \mathrm{~cm}$ ( 6 inches)

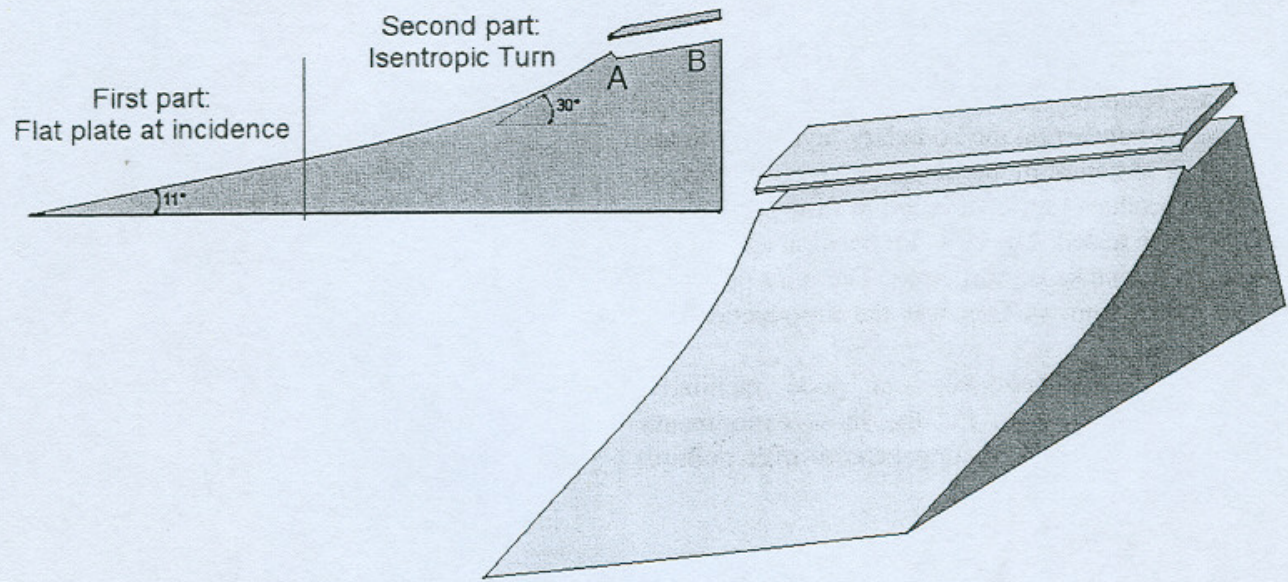

Figure 3. Illustration of the air intake tested. 
Table 1

Flow field characteristics of the Cranfield gun tunnel

\begin{tabular}{cccc}
\multicolumn{3}{c}{$\mathbf{M}_{1}=\mathbf{8} \cdot \mathbf{2}$} \\
$P_{D}$ psig & 2,000 & 1,000 & 500 \\
$p_{0}$ & $1,580 \mathrm{psia}$ & $800 \mathrm{psia}$ & $400 \mathrm{psia}$ \\
& $10 \cdot 89 \times 10^{6} \mathrm{~Pa}$ & $5 \cdot 516 \times 10^{6} \mathrm{~Pa}$ & $2 \cdot 758 \times 10^{6} \mathrm{~Pa}$ \\
$T_{0}$ & $1,290 \mathrm{~K}$ & $1,030 \mathrm{~K}$ & $820 \mathrm{~K}$ \\
$p_{1}$ & $0 \cdot 138 \mathrm{psia}$ & $0 \cdot 0698 \mathrm{psia}$ & $0 \cdot 0349 \mathrm{psia}$ \\
& $951 \cdot 47 \mathrm{~Pa}$ & $481 \cdot 25 \mathrm{~Pa}$ & $240 \cdot 63 \mathrm{~Pa}$ \\
$T_{1}$ & $89 \cdot 3 \mathrm{~K}$ & $71 \cdot 3 \mathrm{~K}$ & $56 \cdot 8 \mathrm{~K}$ \\
$\rho_{1}$ & $0 \cdot 0371 \mathrm{~kg} / \mathrm{m}^{3}$ & $0.0235 \mathrm{~kg} / \mathrm{m}^{3}$ & $0.0148 \times \mathrm{kg} / \mathrm{m}^{3}$ \\
$u_{1}$ & $1,553 \mathrm{~ms}$ & $1,388 \mathrm{~ms}^{-1}$ & $1,239 \mathrm{~ms}$ \\
$\mu_{1}$ & $6 \cdot 368 \times 10^{-6} \mathrm{~Pa} \cdot \mathrm{s}$ & $5 \cdot 104 \times 10^{-6} \mathrm{~Pa} \cdot \mathrm{s}$ & $4.031 \times 10^{-6} \mathrm{~Pa} \cdot \mathrm{s}$ \\
$\mathrm{Re}_{1} /$ inch & $2 \cdot 88 \times 10^{5}$ & $1.618 \times 10^{5}$ & $1.148 \times 10^{5}$ \\
$/ \mathrm{cm}$ & $0.905 \times 10^{5}$ & $0 \cdot 639 \times 10^{5}$ & $0.454 \times 10^{5}$
\end{tabular}

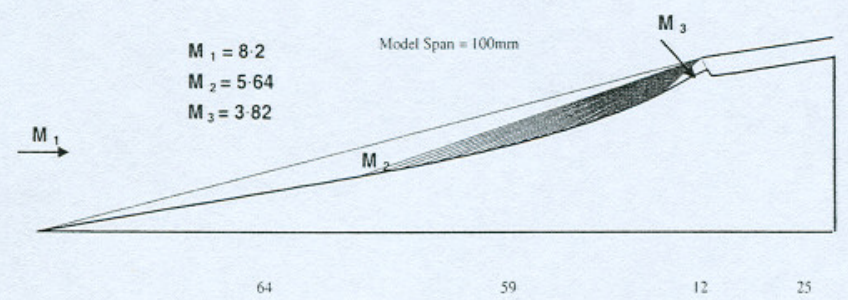

Figure 4. Plot of wave patterns on the intake profile from inviscid values and model dimensions in $\mathrm{mm}$.

diameter, only the $M=8.2$ nozzle was used in these tests giving a running time of around 25 milli seconds.

The working section is of the open jet type with a $25 \mathrm{~cm}$ (10inch) diameter diffuser, all encased in a rectangular sided, square section box coupled to a very large dump tank. The secondary diaphragm enables the dump tank, test section and nozzle to be evacuated to a very low pressure to ensure a quick start to the test flow. Further details of the tunnel and its calibration are given in Refs 2 and 3 . Table I summarises the test conditions of the Cranfield Gun Tunnel used during the experimental studies in this project.

\subsection{Model}

The model tested was a quasi-isentropic intake based on an $11^{\circ}$ wedge followed by an isentropic compression ramp. The turning angle of the flow at the ramp end is $30^{\circ}$.

Several changes have been made to the SHyFE air intake in order to control the flow behaviour in the entrance of the intake. In particular the reflected shock from the cowl inner surface (shown in its simplest form in Fig. 4) can cause boundary layer separation along the floor surface (AB. Fig. 4) downstream of the intake entrance. This separation can spread forwards onto the wedge compression surface and severely disrupt the intake flow. To 'lock'

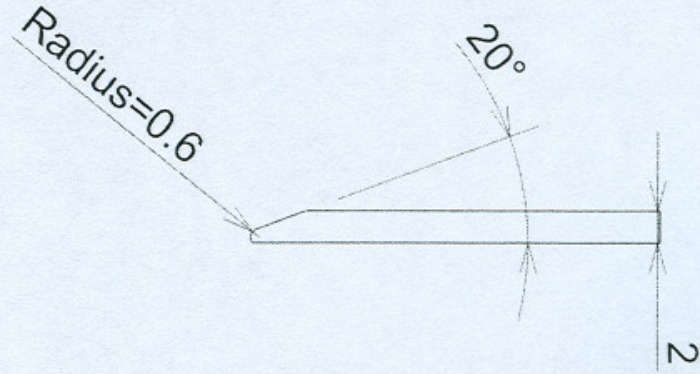

Figure 5. Cowl lip dimensions (in $\mathrm{mm}$ ).

the separation in place and prevent any forward movement an abrupt expansion or dump was added at the throat of the intake.

The position and interaction of shock wave and compression waves formed by the ramp were calculated from the method of characteristics assuming inviscid flow and assuming a zero thickness flat plate cowl. An example of the flow pattern calculated for $\mathrm{M}=$ 8.2 is shown in Fig. 4. The intake has been designed so that at $\mathrm{M}=$ 8.2 all the waves converge to a point which coincides with the leading edge of the cowl.

\subsubsection{Details of the real cowl}

The cowl was formed from a flat plate with a thickness of $2 \mathrm{~mm}$. The leading edge of the cowl is a quarter of cylinder with a radius of $0.6 \mathrm{~mm}$ blending to an external chamfer of $20^{\circ}$ as shown in fig. 5 .

\subsubsection{Vortex generator details}

For the turbulent boundary layer tests vortex generators were placed near the leading edge to force transition. The location and size of the vortex generators are important factors.

According to previous work (Refs 4,5 and 6 ), the vortex generators need to be approximately the size of the boundary layer 


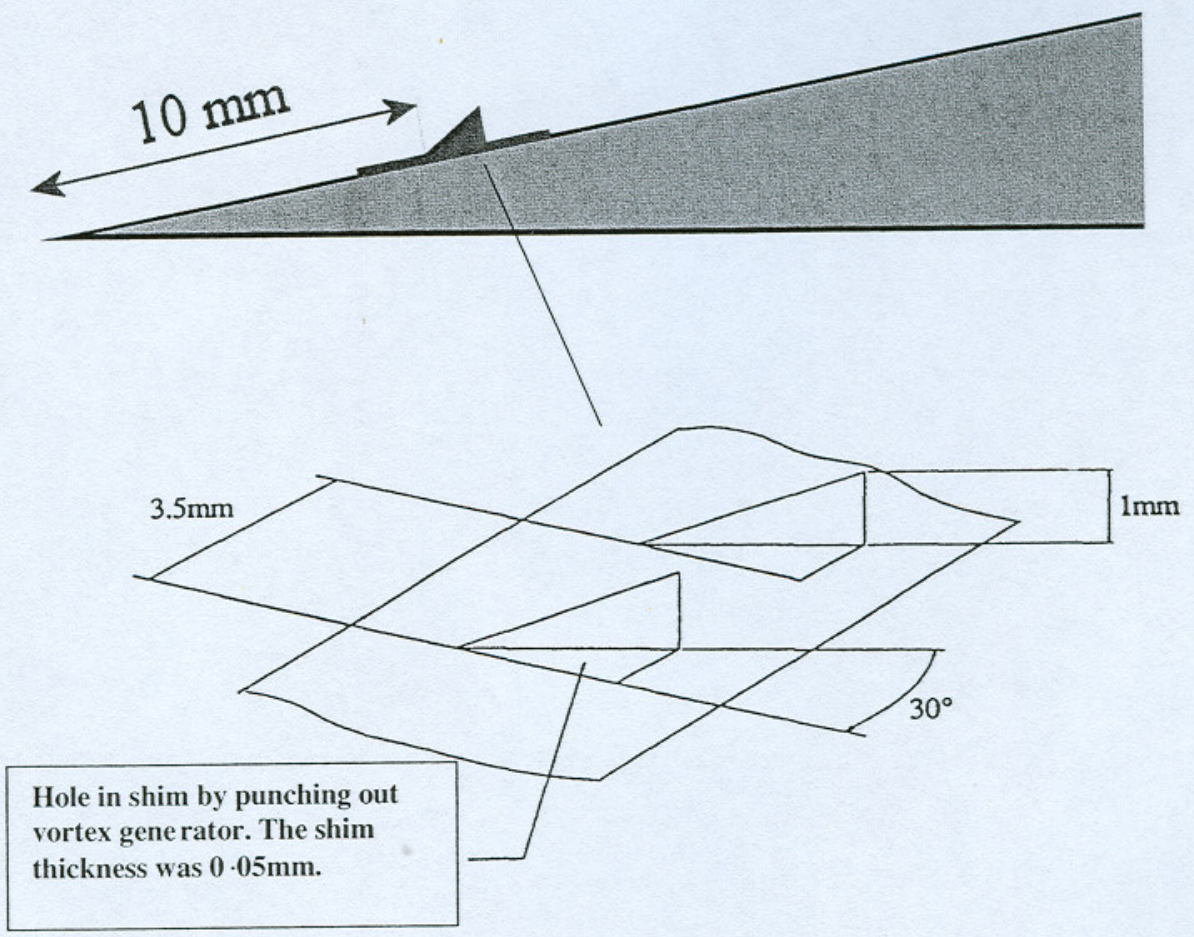

Figure 6. Configuration of the vortex generators.

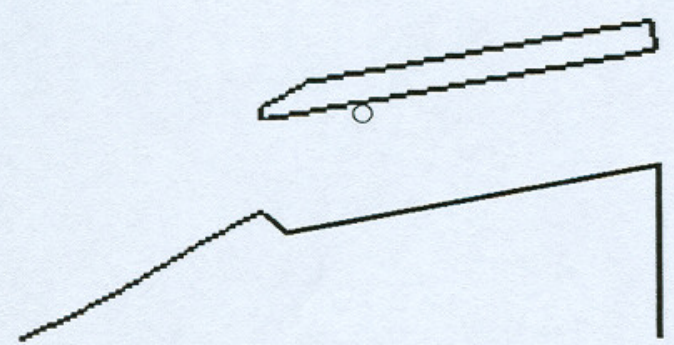

Figure $7(\mathrm{a})$. Wire of $1 \mathrm{~mm}$ diameter.
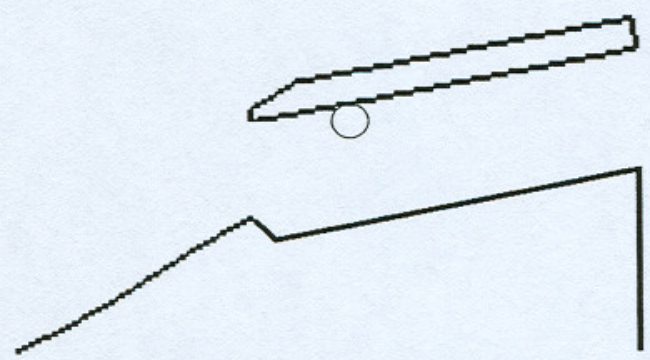

Figure $7(\mathrm{~b})$. Wire of $2 \mathrm{~mm}$ diameter.

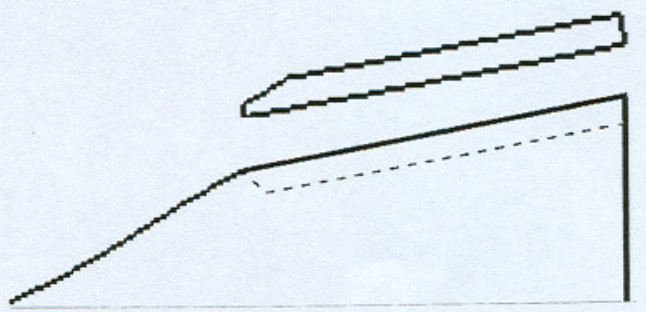

Figure 7(c). Gap filled in. The gap depth was $1.6 \mathrm{~mm}$.

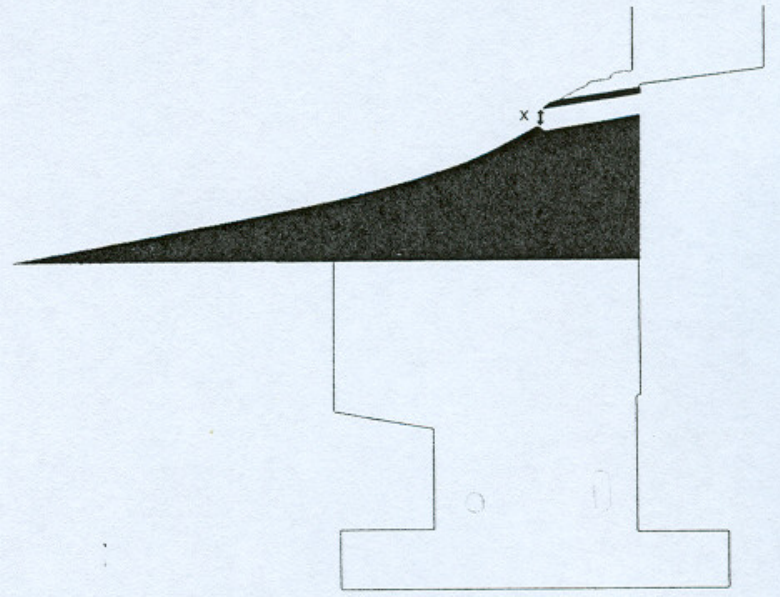

Figure 8. Experimental test configuration.

thickness. Thus, the location and size of the vortex generators were chosen after the analysis of the laminar flow pictures.

They consist of a single row of delta wings $1 \mathrm{~mm}$ high and $3.5 \mathrm{~mm}$ apart. The strip was positioned $10 \mathrm{~mm}$ from the leading edge of the ramp. The delta shaped vortex generators were inclined at $30^{\circ}$ to the flow direction as shown in Fig. 6 .

\subsubsection{Modifications to the internal flow}

The effect of geometric restrictions on the intake flow starting process was investigated by introducing a wire on the upper wall of the throat (cf. Figs 7(a) and 7(b) and by filling up the gap behind the step on the lower wall of the duct (Fig. 7(c)). The wires were placed $5 \mathrm{~mm}$ from the cowl lip. 


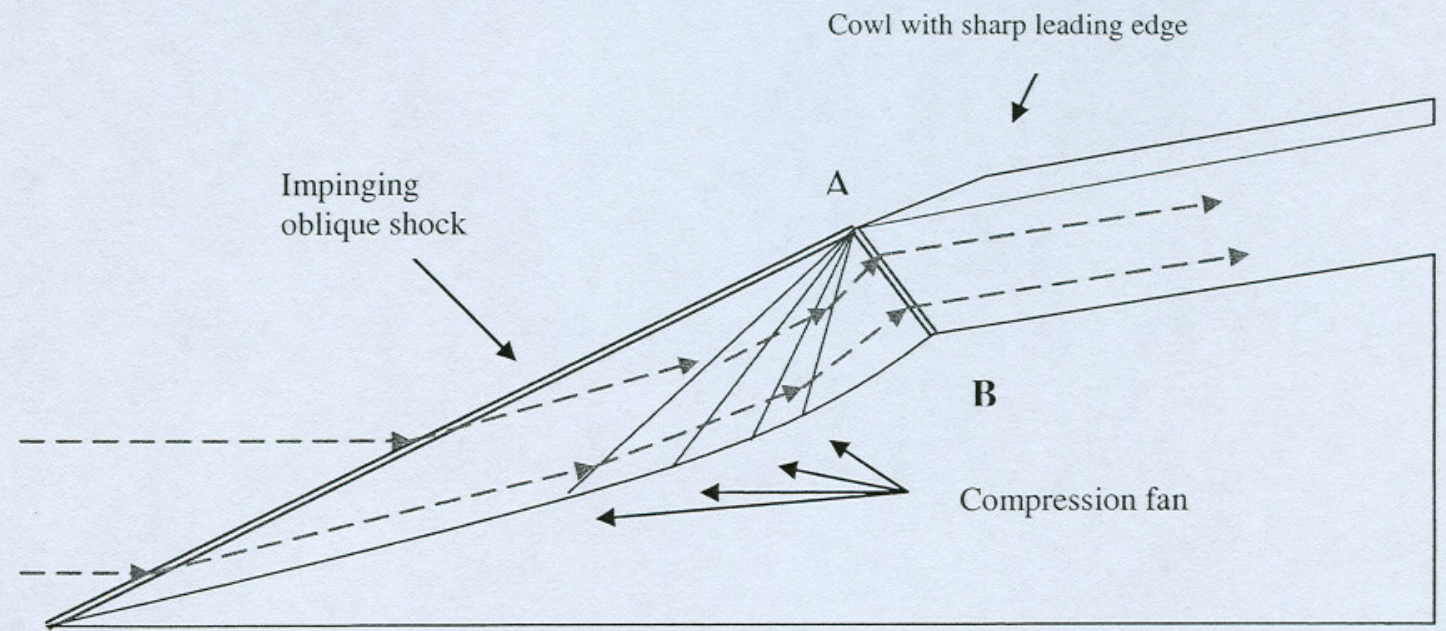

Figure 9(a). Ideal inviscid flow, fully attached flow.

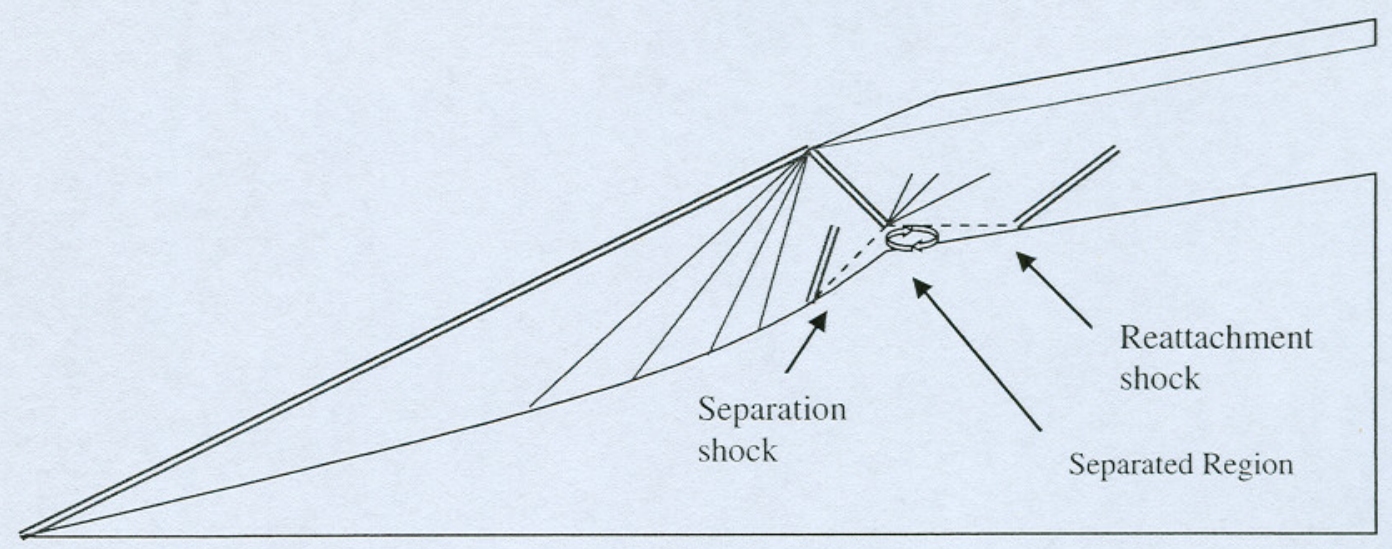

Figure. 9(b).Viscous flow. Reflected shock causing separation at the intake entry.

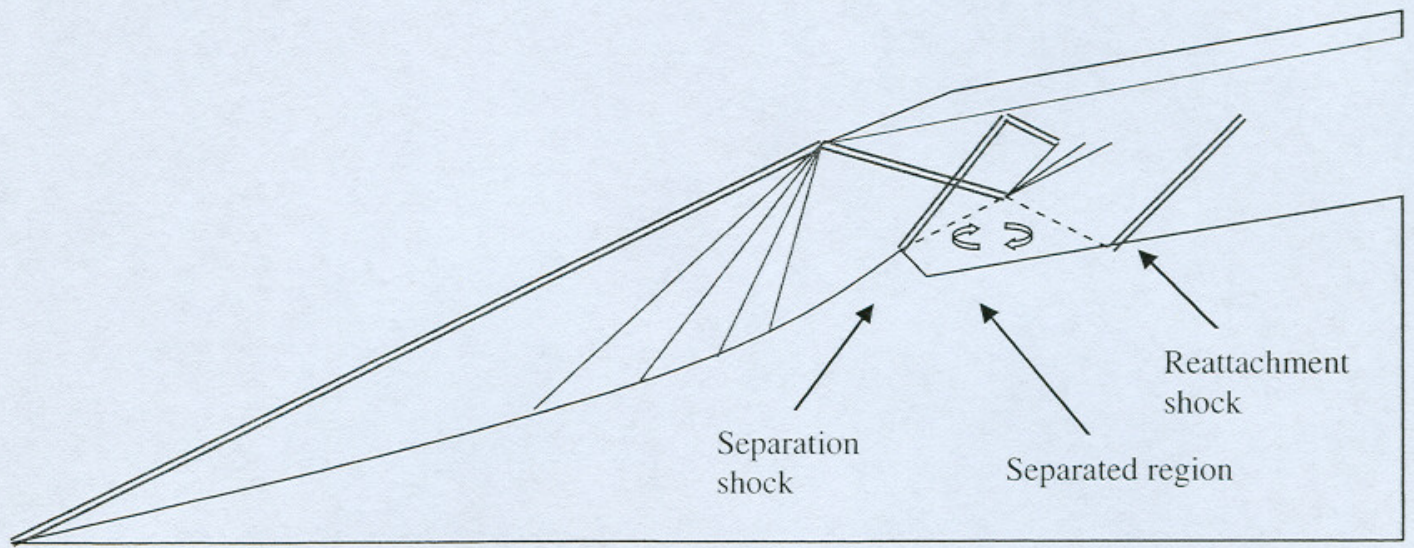

Figure 9(c). The gap or 'dump' at the entry to the intake prevents the separated region extending forwards onto the ramp surface. 


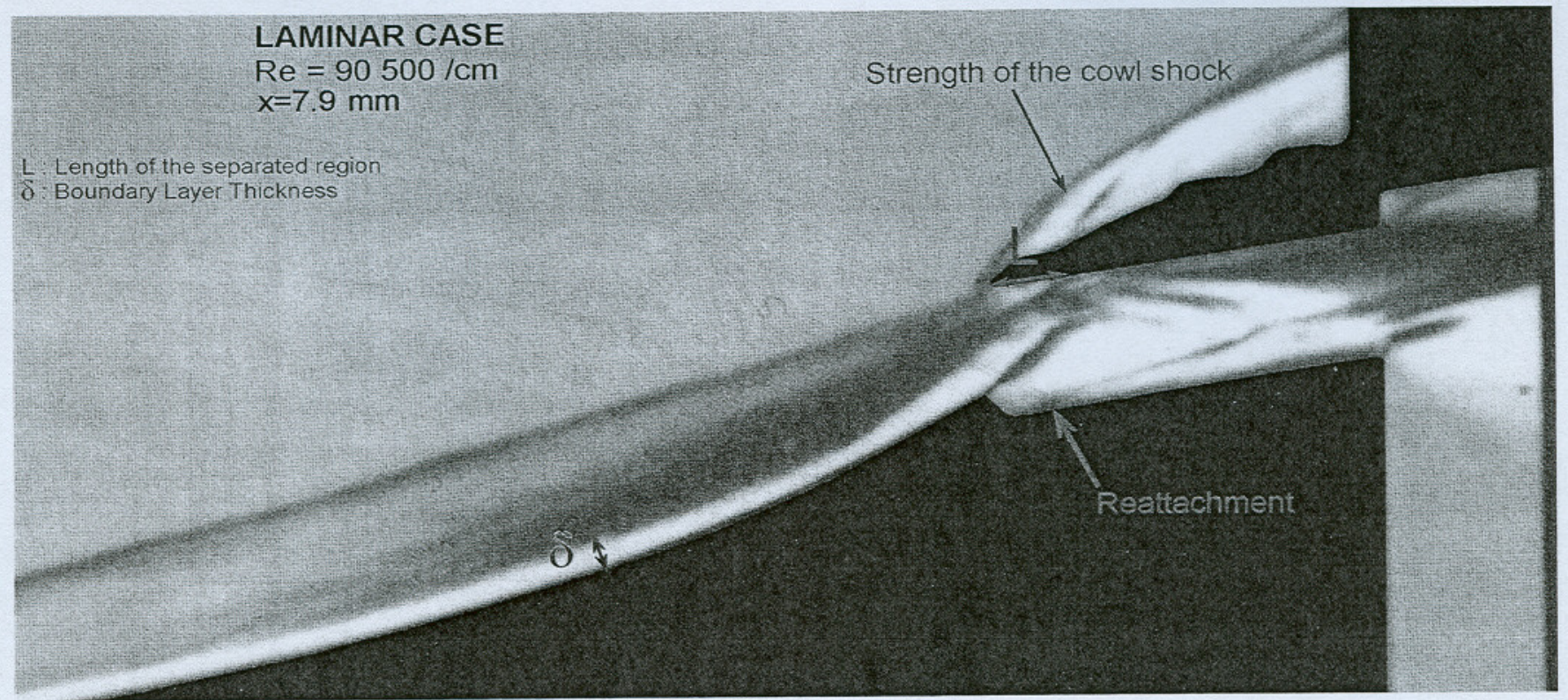

Figure 10(a). Flow characteristics measured for the analysis.

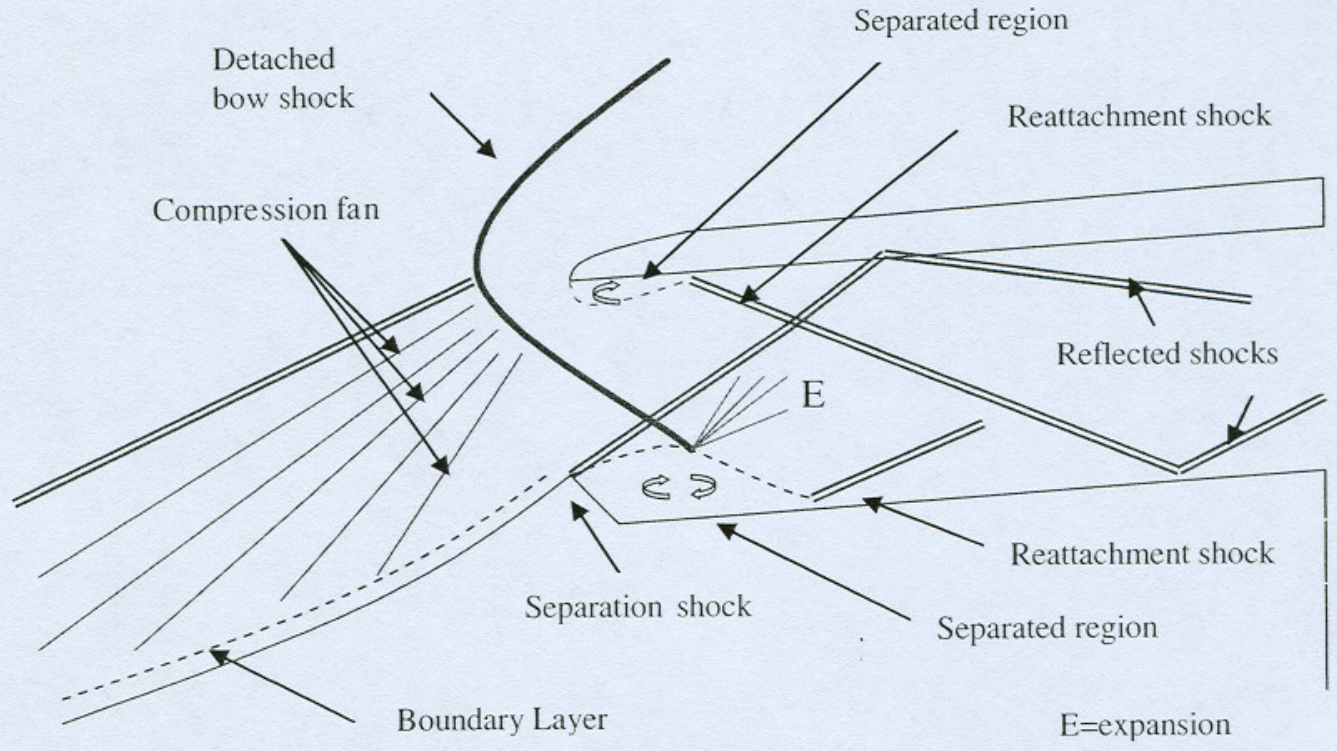

Figure 10(b). Main features of the flow shown in Fig 10(a)

Table 2

Cowl lip place influence

Gun Tunnel operating condition

\section{Model Configuration}

Figure No

Cowl lip position $x$

Slope of the cowl lip shock $\theta$

Length of the separated

region (real value) $L$

$$
\begin{gathered}
\mathrm{M}_{1}=8 \cdot 2 \\
\operatorname{Re}^{\mathrm{I}}=\mathbf{9 0 , 5 0 0 / \mathrm { cm }}
\end{gathered}
$$

Laminar boundary layer

$\begin{array}{cccc}\text { Fig. } 11 & \text { Fig. } 12 & \text { Fig. } 13 & \text { Fig. } 14 \\ 4.3 \mathrm{~mm} & 6.58 \mathrm{~mm} & 7.9 \mathrm{~mm} & 8.32 \mathrm{~mm} \\ 57^{\circ} & 47^{\circ} & 39^{\circ} & 37.5^{\circ} \\ \text { none } & \text { none } & 4.35 \mathrm{~mm} & 8.26 \mathrm{~mm}\end{array}$




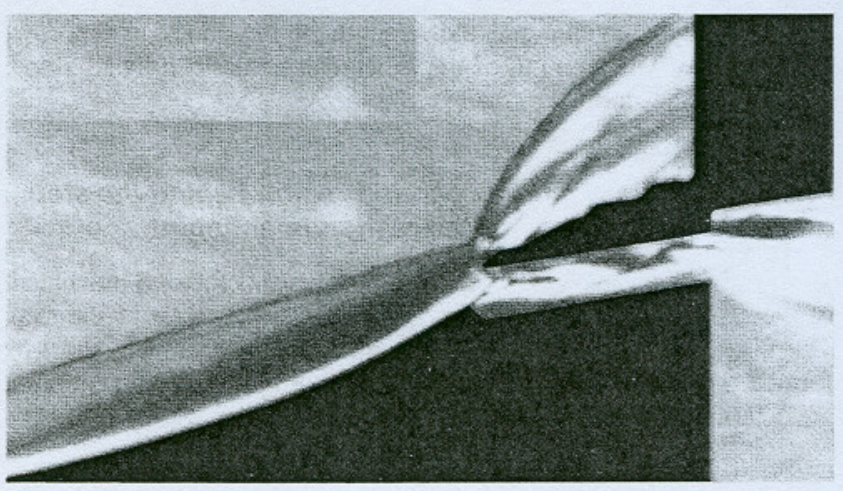

Figure 11. $x=4 \cdot 3 \mathrm{~mm}$.

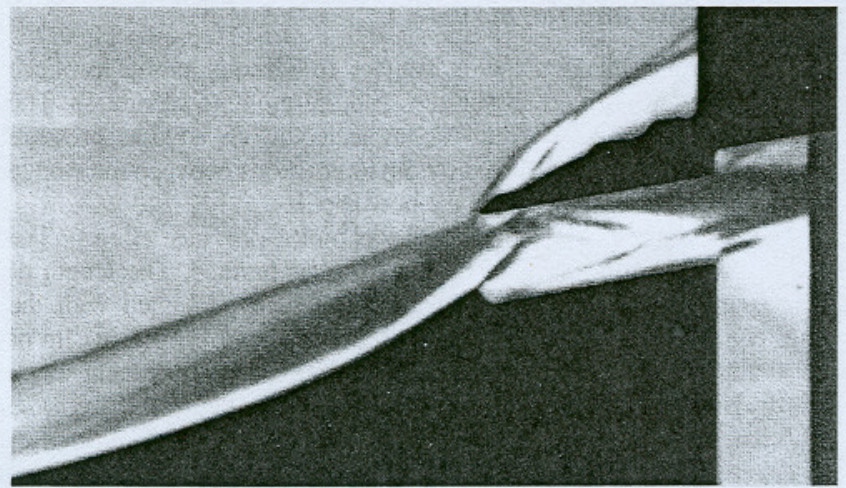

Figure 13. $x=7 \cdot 9 \mathrm{~mm}$

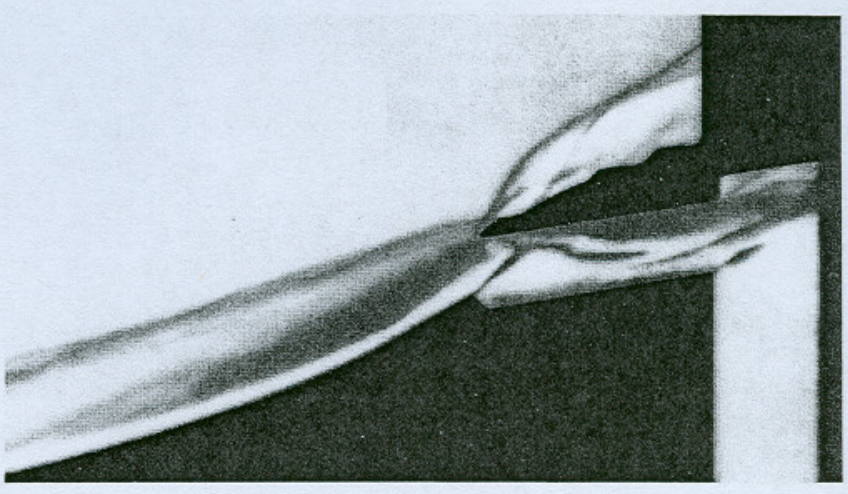

Figure 12. $x=6 \cdot 58 \mathrm{~mm}$.

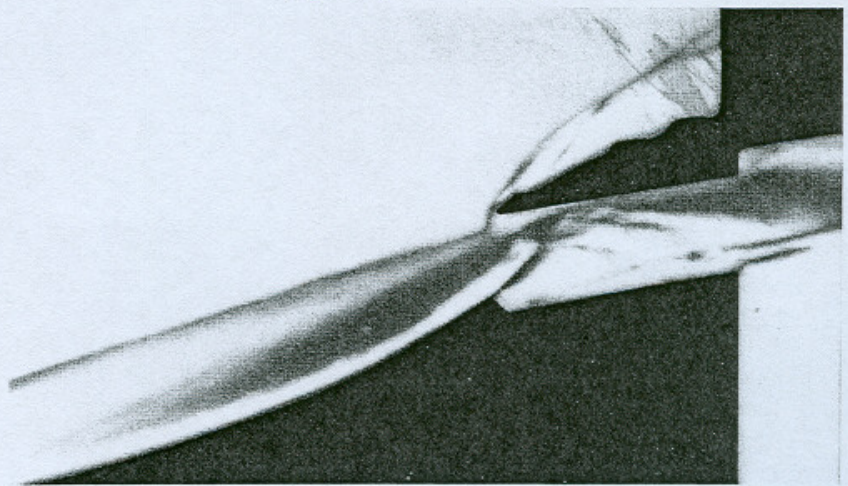

Figure 14. $x=8 \cdot 32 \mathrm{~mm}$

Cowl lip place influence: Laminar case, $R e_{1}=90,500 / \mathrm{cm}$ and $M_{1}=8 \cdot 2$.

\subsection{Schlieren system}

To obtain pictures of the flow pattern, high speed Schlieren photography was employed. The Schlieren system uses a Toepler arrangement using a short duration argon stabilised spark source.

The screen on which the image is projected is part of a Polaroid camera type 667 which uses 300ASA black and white film to capture the image of the flowfield.

Schlieren pictures have been scanned with the highest resolution $(1,200$ pixels/inch) to provide the best accuracy. This gives an accuracy of plus or minus 1 pixel or $0.02 \mathrm{~mm}$ for the scanning process.

Although the Schlieren pictures have been magnified as much as possible, the measurements were done by eye.

\subsection{Set-up}

The set-up of the model in the tunnel is shown in Fig. 8. The ramp is mounted on a pedestal from the tunnel floor. The cowl lip is fixed on a strut by two screws and attached to the tunnel roof.

The cowl lip position can be moved vertically to analyse the influence of the cowl location on the flow behaviour. Therefore, the height of the intake entrance is the variable ' $x$ ' in Fig. 8.

\subsection{RESULTS AND DISCUSSION}

A close examination of any of the Schlieren pictures will show how complex the real flow is. This is because the viscous effects of boundary layer displacement and separation are significant.

The inviscid flow at the design condition is easy to draw, particularly if the cowl lip is sharp (Fig. 9(a)). The reflected shock AB turns the flow back to the horizontal producing a uniform high pressure stream at the intake entry. If however the shock $A B$ separates the boundary layer at the point B then a separation bubble will form as shown in Fig. 9(b). To prevent the separation point moving forward and unstarting the intake a 'dump' has been fitted and the resulting flow pattern should resemble the sketch in Fig.9(c).

In our tests the boundary layer growth along the compression surface means that the leading edge shock and the isentropic fan do not meet in a point. The cowl leading edge is blunted to reduce the local heat transfer rate so there will be shock/shock interactions in that region. Nevertheless many of the features pictured in Fig.9(c) are visible.

\subsection{Presentation}

The general behaviour of the flow is examined on each picture to determine the effects of changes of geometric configuration. The analysis compares the features shown on Fig. 10:

\subsubsection{Evaluation of the boundary layer thickness, $\delta$}

This investigation is only performed for the laminar case due to the difficulty in detecting the boundary layer edge in the turbulent case. The boundary layer is measured from the Schlieren pictures which record the density gradients in the vertical direction since the knife edge was horizontal. In a hypersonic boundary layer the density gradients are small until close to the outer edge. The edge being defined as the point where the inviscid value of the velocity is reached. However the density itself is so low in much of the boundary layer profile that the displacement thickness $\delta^{*}$ is typically $85 \%$ of $\delta$. The values of $\delta$ measured from the pictures are compared with the calculated values of $\delta^{*}$ in section 3.2 .

\subsubsection{Evaluation of the upper cowl shock strength}

The strength of the cowl leading edge shock on the outer surface is an important parameter since it displays the amount of the flow spilling out above the air intake and influences the properties of the flow entering in the air intake. The goal of this investigation is to 


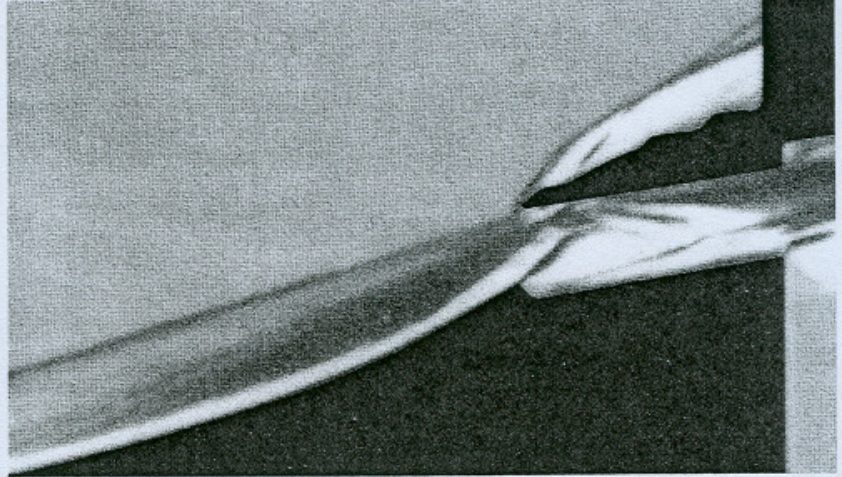

Figure 15. $\operatorname{Re}_{1}=90,500 / \mathrm{cm} \quad x=7 \cdot 9 \mathrm{~mm}$

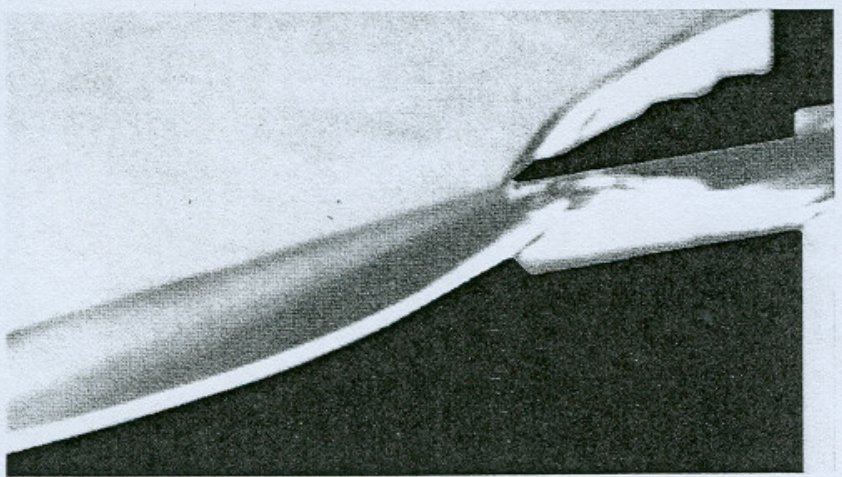

Figure 16. $R \mathrm{Re}_{1}=63,900 / \mathrm{cm} \quad x=7 \cdot 2 \mathrm{~mm}$

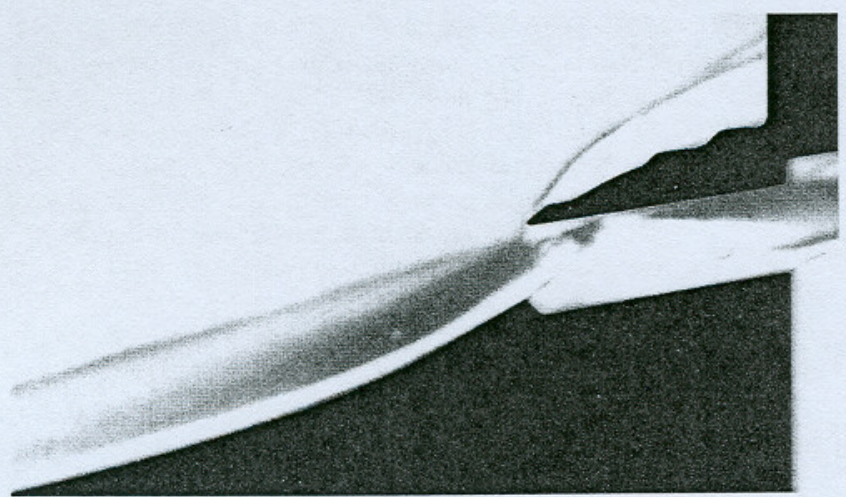

Figure 17. $R e_{1}=45,400 / \mathrm{cm} \quad x=7 \cdot 9 \mathrm{~mm}$

Reynolds Number Influence on the intake entry section with roughly the same cowl position and a Laminar Boundary Layer. compare the behaviour and the strength of the shock at the cowl leading edge. The strength of the shock can be defined by its slope. The greater the slope, the stronger the shock.

\subsubsection{Evaluation of the separated region behind the step and the location of the reattachment point}

The addition of a step at the intake throat prevents forward movement of the separation region which now starts near the crest of the step. The flow reattaches further downstream and a reattachment shock is formed. The length of the separated region and the reattachment point are determined from the enlarged Schlieren pictures.

\subsubsection{Evaluation of the length of the separated region on the cowl internal surface, $L$}

The hypersonic intake flow is subject to viscous problems since boundary layers form on all surfaces and are prone to separation. The leading edge of the cowl can be subjected to an adverse pressure gradient since it can be affected by the interactions between the wedge shock, the compression fan and the bow shock.

Figure 10(a) shows some of the complex viscous effects. The separated region on the cowl inner surface is marked. The length of this separated region $(L)$ is measured and then compared with the other configurations. There is also a separated region from the lip of the 'dump' to a point on the lower surface where a reattachment shock is just visible. The wedge shock and compression fan reflect from the cowl inner surface but will be white regions and are therefore difficult to see. Our interpretation of the flow is shown in fig. 10(b)

\subsection{Effect of cowl position for laminar flow (Figs 11-14)}

The results from Table 2 show that the cowl upper shock decreases in strength as the cowl is moved further away from the ramp. Figure 11 shows the case where the cowl is nearest to the ramp and where the cowl shock is the strongest. In this case much of the flow is going outside the intake entrance. Thus the intake is starting but spilling much of the flow and the cowl drag will be high.

In Figs 11 and 12 the wedge shock is passing upstream of the cowl leading edge and in this case no sign of separated flow can be seen. Figs 13 and 14 show a wedge shock passing downstream of the leading edge and the boundary layer separates because of the sudden pressure step due to the wedge shock and the centred compression fan. This causes an adverse pressure gradient which separates the flow. In addition, it should be noted that in the case when the wedge shock and compression fan impinges further downstream of the cowl leading edge (i.e. for the largest intake entry section Fig. 14), the separation length is significantly greater.

It is also clear that the greater the height of the intake entrance is, the smaller the separated region is behind the step in the throat. In addition, it is immediately noticeable that the vertical extent of

Table 3

Reynolds number influence

Tunnel Gun operating : condition

Model Configuration:

Figure No

Cowl lip position $x$

Slope of the cowl lip shock $\theta$

Length of the separated

region (real value) $L$

$$
\mathrm{M}_{1}=8 \cdot 2
$$

$\mathrm{Re}_{1}=63,900 / \mathrm{cm}$

$\operatorname{Re}_{1}=45,400 / \mathrm{cm}$

Laminar boundary layer

Fig. 16
$7.2 \mathrm{~mm}$
$41^{\circ}$
$3.7 \mathrm{~mm}$

Fig. 17

$7.9 \mathrm{~mm}$

$42.5^{\circ}$

$6.2 \mathrm{~mm}$

$3.7 \mathrm{~mm}$
Fig. 15

$7.9 \mathrm{~mm}$

$4.6 \mathrm{~mm}$ 


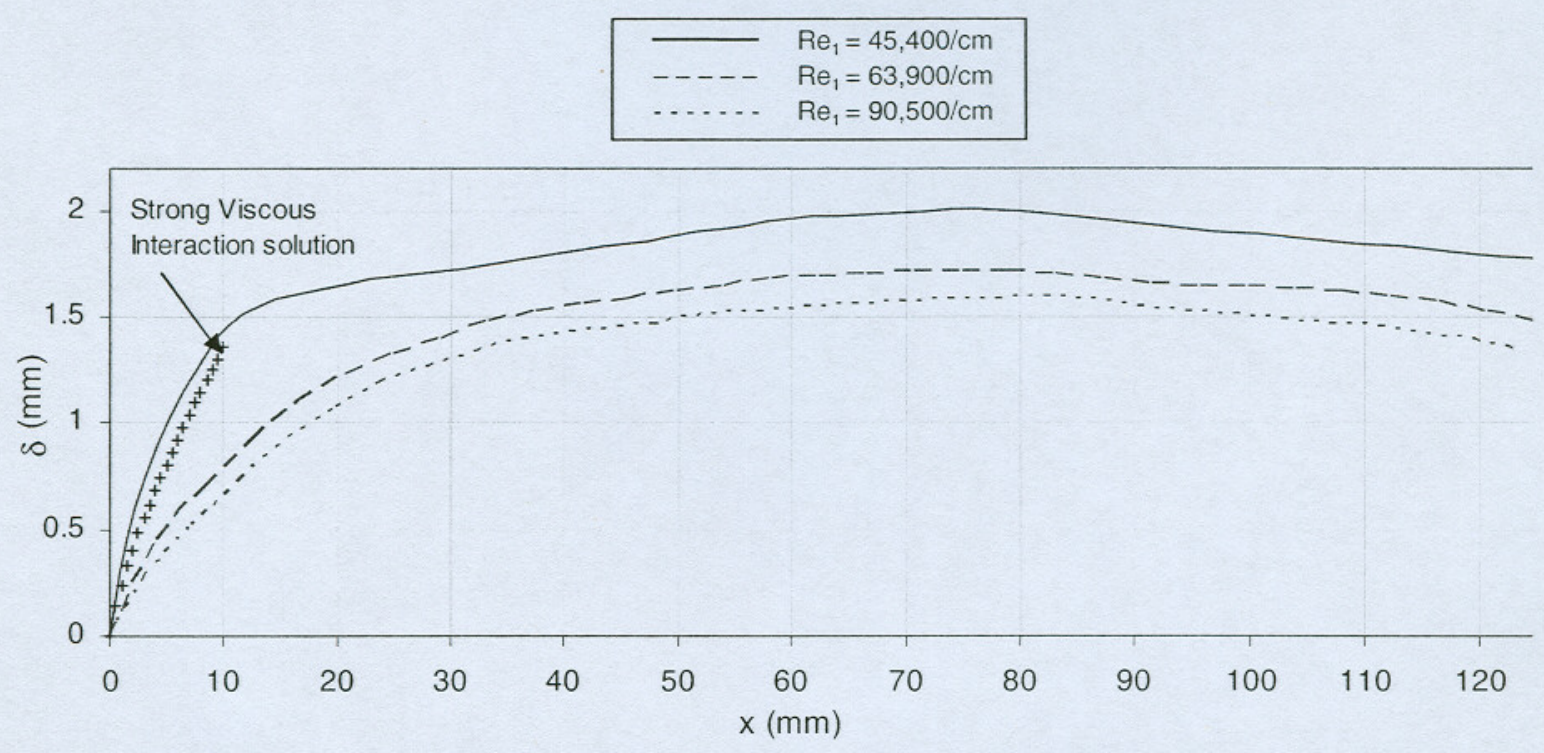

Figure 18. Influence of the Reynolds number on the boundary-layer thickness measured from Figs 15,16 and 17.

this separated region is far more pronounced than when the cowl position is close to the ramp. It could be concluded that the higher the cowl lip, the more flow enters the intake, the greater the pressure at the intake entry and hence the reduction in the separated region on the lower intake surface.

\subsection{Effect of the Reynolds number in the laminar case (Figs 15-17)}

The boundary layer thickness along the ramp is measured from figs 15 to 17 and plotted on fig 18 .

The Reynolds number influence on the boundary layer thickness is in good agreement with that predicted by the theory. It can be seen that the boundary layer growth decreases as the Reynolds number rises. In addition, the same tendency is observed for all Reynolds numbers: an increase of the boundary layer thickness on the first part of the ramp corresponding to a flat plate at incidence and a slight decrease through the isentropic turn. The surface curvature stops at a value $x=125 \mathrm{~mm}$ (point $A$ shown in Fig. 19). The end of the ramp after the point $\mathrm{A}$ can be equivalent to a flat plate at $30^{\circ}$ of incidence with uniform flow. However it is impossible to see the edge of the boundary layer in this region. The point $\mathrm{A}$ is the limit of the measurements of $\delta$.

Very near the leading edge there is a strong viscous interaction. Using the local flat plate similarity solution for hypersonic boundary layer growth and the tangent wedge rule for the pressure distribution

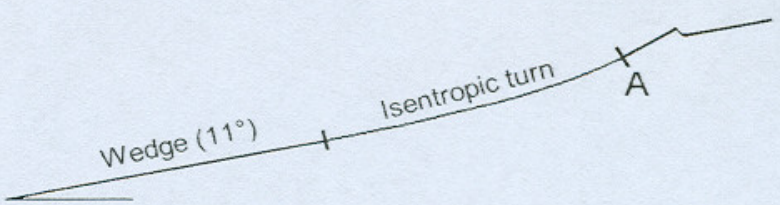

Figure 19. Curvature surface drawing of the compression ramp.

it can be shown that (Ref. 7) the boundary layer displacement thickness is given by:

$$
\begin{aligned}
& \qquad \mathrm{M}_{\infty} \frac{\delta^{*}}{x}=\left(\frac{2 A \bar{\chi}}{B}\right)^{1 / 2} \\
& \text { where } A=0 \cdot 664\left(\frac{\gamma-1}{2}\right)\left(1+2 \cdot 6 \frac{T_{\mathrm{W}}}{T_{0}}\right) \text { and } B=3 / 4[\gamma(\gamma+1)]^{1 / 2} \\
& \bar{\chi}=\mathrm{M}_{\infty}^{3} \sqrt{\frac{\mathrm{C}}{\mathrm{Re}_{x}}} \text { Laminar viscous interaction parameter }
\end{aligned}
$$

The result for $\mathrm{Re}_{1}=45,400 / \mathrm{cm}$ is marked on Fig. 18 in the region where it applies. Further along the surface the effects of incidence, "i.e. wall slope', dominate the boundary layer growth.

Table 4

Effect of vortex generators at three Reynolds numbers

\begin{tabular}{|c|c|c|}
\hline \multirow[b]{2}{*}{$\begin{array}{c}\text { Model Configuration: } \\
\text { Figure No }\end{array}$} & \multicolumn{2}{|c|}{$\mathrm{Re}_{1}=90,500 / \mathrm{cm}$} \\
\hline & $\begin{array}{l}\text { turbulent } \\
\text { Fig. } 20\end{array}$ & $\begin{array}{c}\text { laminar } \\
\text { Fig. } 21\end{array}$ \\
\hline Cowl lip position $x$ & $7.8 \mathrm{~mm}$ & $7.9 \mathrm{~mm}$ \\
\hline & $39^{\circ}$ & $39^{\circ}$ \\
\hline ength of the separated & $5 \mathrm{~mm}$ & $4.6 \mathrm{~mm}$ \\
\hline
\end{tabular}

* The Schlieren pictures at the two lower Reynolds numbers are so similar that they are not included

Gun Tunnel operating condition:

region (real value) $L$

$$
\mathrm{M}_{\infty}=8 \cdot 2
$$

$\operatorname{Re}_{1}=63,900 / \mathrm{cm}$

turbulent lamina

Not shown Not shown

$\begin{array}{cc}7.8 \mathrm{~mm} & 7.2 \mathrm{~mm} \\ 39^{\circ} & 41^{\circ} \\ 6 \mathrm{~mm} & 3 \mathrm{~mm}\end{array}$

$\operatorname{Re}_{1}=45,400 / \mathrm{cm}$

turbulent lamina

Not shown Not shown

$7.8 \mathrm{~mm} \quad 7.9 \mathrm{~mm}$

$41^{\circ} \quad 42.5^{\circ}$

$5.6 \mathrm{~mm} \quad 6.2 \mathrm{~mm}$ 


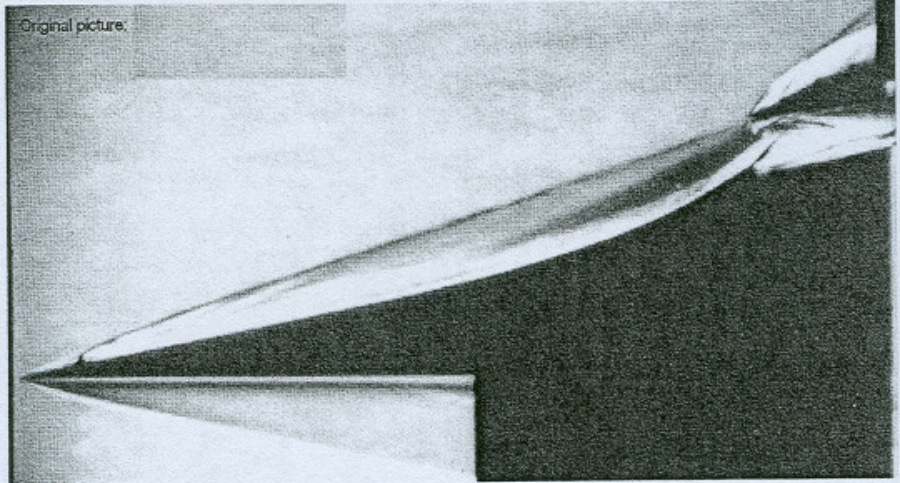

Figure 20. Turbulent $\mathrm{BL} x=7 \cdot 8 \mathrm{~mm}$.

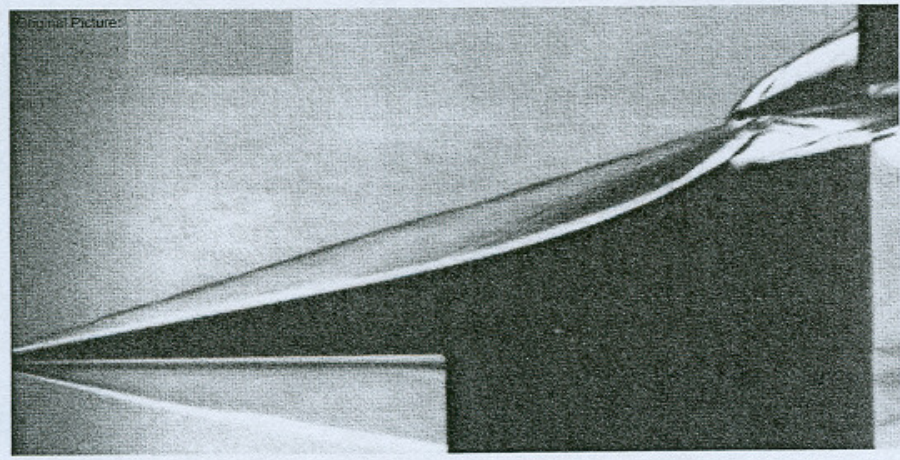

Figure. 21. Laminar $\mathrm{BL} x=7.9 \mathrm{~mm}$.

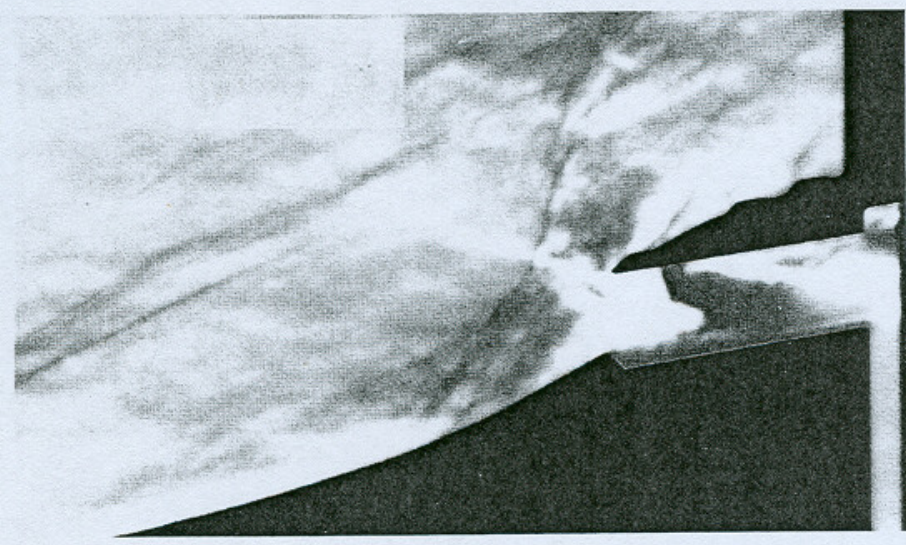

Figure 22. Wire of $2 \mathrm{~mm}$ of diameter, $x=8.2 \mathrm{~mm}$. Turbulent boundary layer.

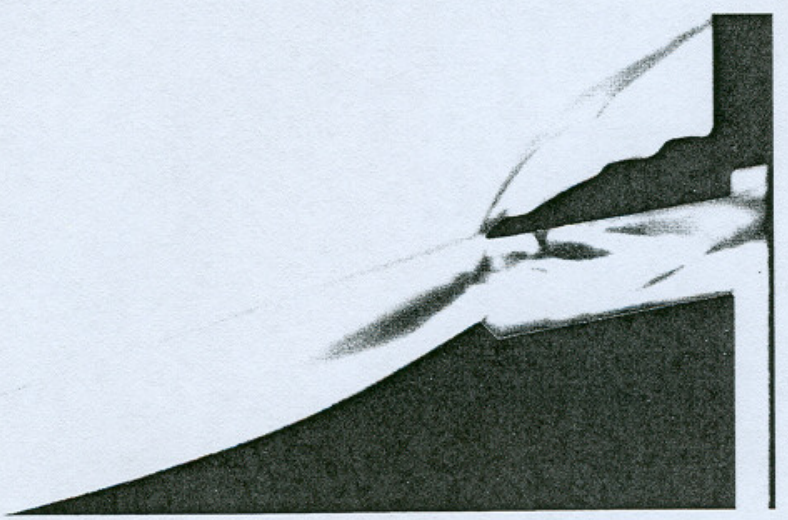

Figure 23. Wire of $1 \mathrm{~mm}$ of diameter, $x=8.2 \mathrm{~mm}$. Turbulent boundary layer
Table 3 shows that Reynolds number has an effect on the strength of the shock generated by the cowl leading edge and the shock strength appears to increase with decreasing Reynolds number. This is probably due to the increased boundary-layer displacement thickness causing more of the intake flow being spilt around the top of the cowl.

However the difference between the slope angles is small so the actual rank given must be treated with caution. Unfortunately, the run at $\mathrm{Re}_{1}=$ $63,900 / \mathrm{cm}$ has an intake entrance height slightly smaller than the other cases. Thus it influences the results.

Concerning the separated region behind the step on the lower surface it is difficult to detect the reattachment point in the case when $\mathrm{Re}_{1}=$ $63,900 / \mathrm{cm}$ whereas for the other cases $\left(\operatorname{Re}_{1}=90,500 / \mathrm{cm}\right.$ and $\left.45,400 / \mathrm{cm}\right)$, the separated region length and the location of the reattachment seem to be approximately similar.

The separation of the flow on the upper wall of the intake throat can be seen at all Reynolds numbers.

The length of flow separation associated with the three Reynolds numbers is presented in Table 3. A comparison can be made between the cases with the same height entrance, i.e. Figs 23 and 25 . The results indicate clearly that the Reynolds number affects the separated region length. The lower the Reynolds number the greater the separated region length seems to be. This again is probably due to viscous interaction near the wedge leading edge. Lowering the Reynolds number increases the boundary-layer displacement thickness and hence the shock strength.

\subsection{Effect of vortex generators (Figs 20-21)}

In this section, a comparison is made between laminar and turbulent boundary layers at each Reynolds number.

Figs 20 and 21 present the effect of utilizing vortex generators placed near the leading edge. Two Schlieren pictures are shown at the same Reynolds number with roughly the same cowl lip position, one with vortex generators and the other without.

It can be seen by comparing the two pictures that the thickness of the boundary layer appears to be significantly greater with the vortex generators. The pictures suggest a boundary layer thickness of $1.45 \mathrm{~mm}$ in the laminar case and $3.1 \mathrm{~mm}$ in the turbulent case at a position of $116 \mathrm{~mm}$ from the leading edge of the ramp.

All values of the cowl shock slope measured are grouped in Table 4 to compare the laminar and turbulent cases at every Reynolds number In view of the small differences between the angle values; it is impossible to draw any conclusion. Similarly nothing significant can be said about the comparison of the separated region length.

The influence of tripping the boundary layer on the separation behind the step and on the internal surface of the cowl is negligible. The most interesting fact is how little difference the vortex generators have made to the overall flow except to the boundary layer thickness.

\subsection{Effect of geometric restrictions (Figs 22-25) turbulent} flow

Two types of restrictions were added in the intake throat. Figures 22 and 23 present the effect of placing a wire on the upper wall of the throat and Figs 24 and 25 show the effect of filling in the step on the lower wall of the duct.

An interesting comparison can be made in Figs 22 and 23 between the introducing of a $2 \mathrm{~mm}$ and $1 \mathrm{~mm}$ wire. Schlieren pictures indicate that the air intake has started in the case of a $1 \mathrm{~mm}$ diameter wire unlike the case with a $2 \mathrm{~mm}$ diameter wire.

Figures 24 and 25 show the effect of the step at the throat for a turbulent boundary layer. The intake starts perfectly well without the step. The only difference between the pictures is the stronger cowl shock in Fig: 24 and the more outboard position of the wedge shock. These effects are probably due to the reduced intake area downstream of the lip.

To conclude, the effect of solid blockage is clear on the intake flow starting process. The experiments show the significant sensitivity of the flow to blockage effects since the intake has been started with the introduction of a $1 \mathrm{~mm}$ diameter wire on the inner cowl surface whereas with the $2 \mathrm{~mm}$ diameter wire the intake has been unable to start. 


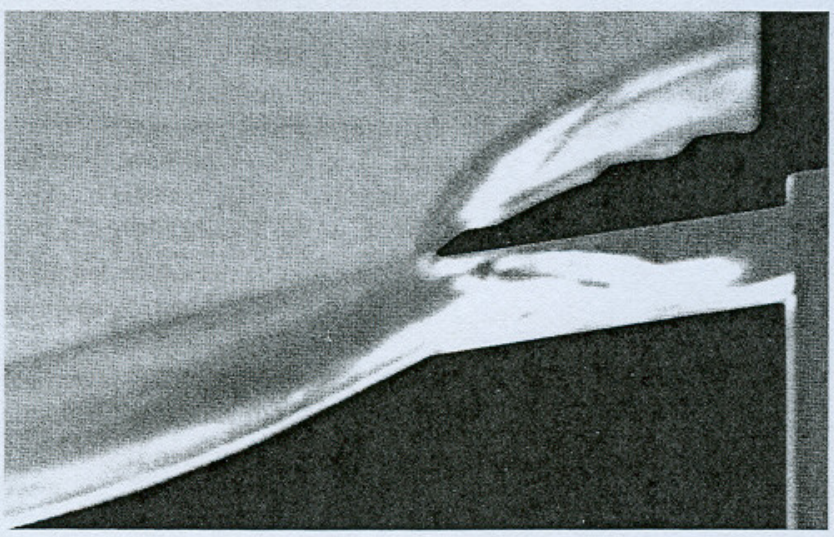

Figure 24. Gap behind the step filled in $x=8.1 \mathrm{~mm}$. Turbulent BL.

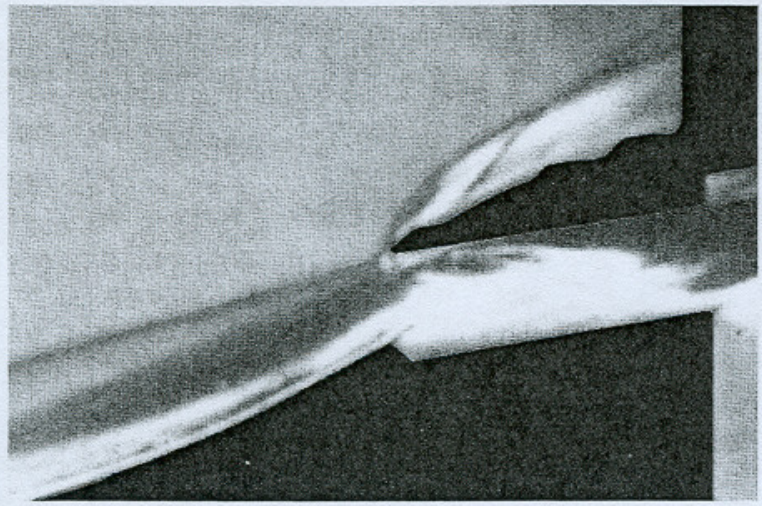

Figure 25. Presence of the step at the throat, $x=7 \cdot 8 \mathrm{~mm}$. Turbulent BL.

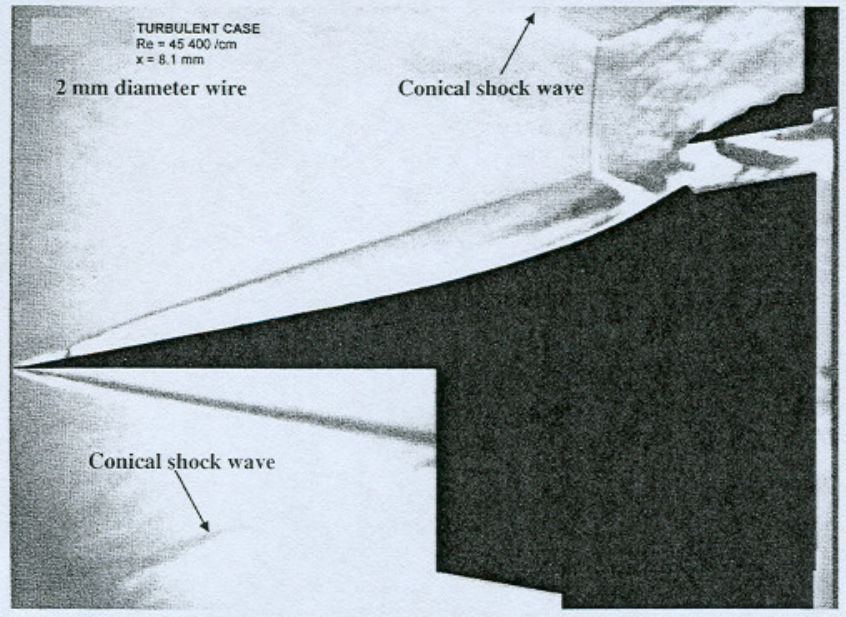

Figure 26. Blocked gun tunnel working section and example of an 'unstarting' intake. $x=8.1 \mathrm{~mm} \mathrm{Re}{ }_{1}=45,400 / \mathrm{cm}$

The reason why a $2 \mathrm{~mm}$ diameter wire 'blocks' the intake whereas the filling - in of a $1.6 \mathrm{~mm}$ step does not is probably due to the wire reducing the entry distance $x$ (fig. 8 ) and the fact that the boundary layer on the ramp surface is turbulent. It would be interesting to repeat the comparison for a laminar boundary layer since tests elsewhere have shown that exntensive flow separation can unstart the intake (Ref. 1).

Figure 26 illustrates the flow behaviour around the air intake which was subject to a blockage effect. This is due to the introduction of the $2 \mathrm{~mm}$ diameter wire and insufficient nozzle pressure ratio which creates a tunnel blockage as shown in this figure.

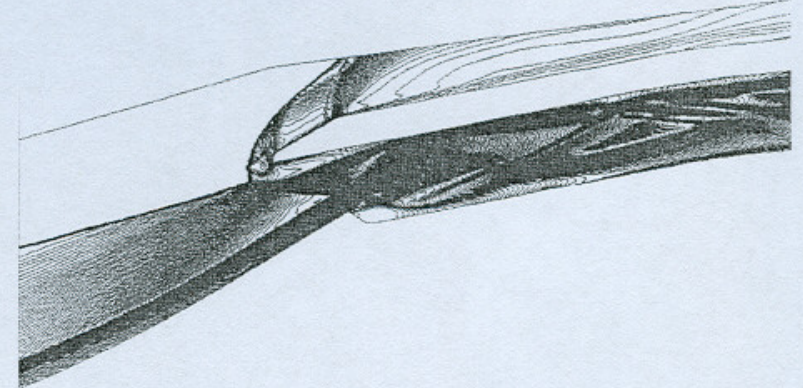

Figure 27. $M_{1}=6$ and $R e_{1}=55,000 / \mathrm{cm}$.

From Prof R. Hillier, Imperial College London

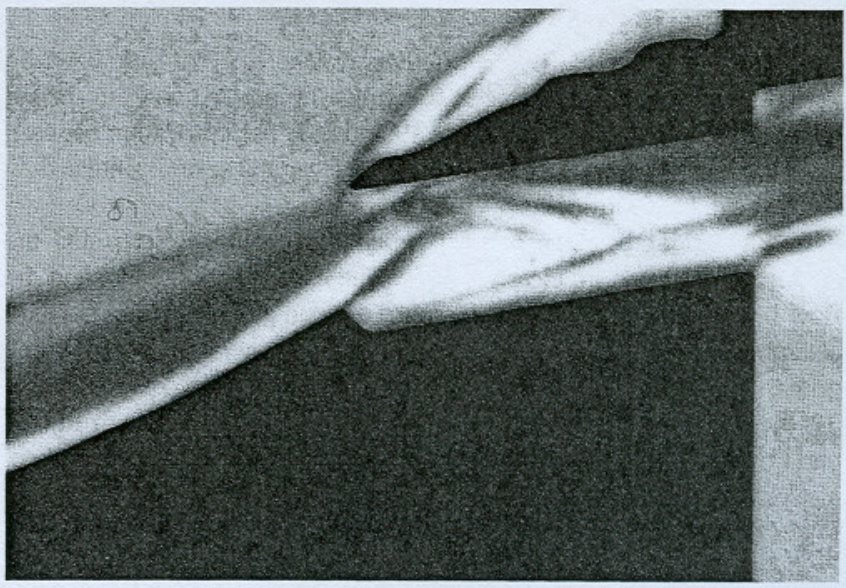

Figure 28. $M_{1}=8 \cdot 2$ and $R_{1}=90,500 / \mathrm{cm}$

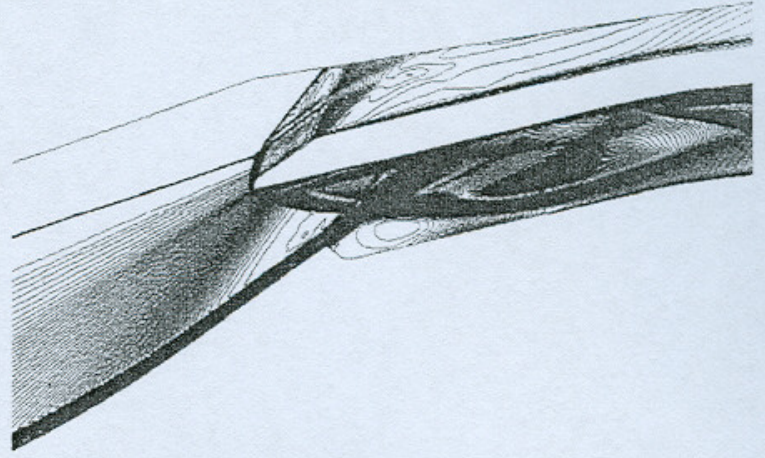

Figure 29. $M_{1}=5$ and $\operatorname{Re}_{1}=55,000 / \mathrm{cm}$.

(From Prof R. Hillier, Imperial College London)

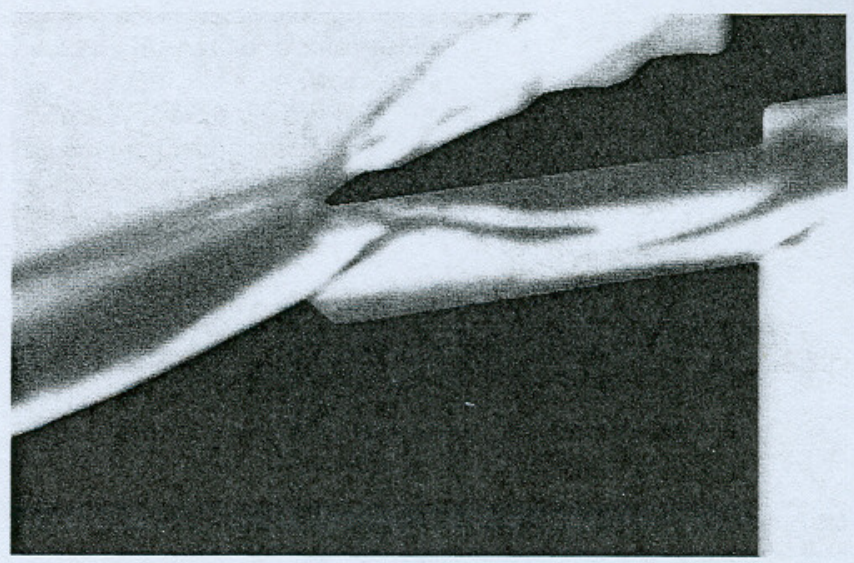

Figure 30. $M_{1}=8 \cdot 2$ and $R e_{1}=90,500 / \mathrm{cm}$. 
The flow at the exit of the tunnel nozzle can be expanded to a lower pressure level than the pressure that surrounds the test section. The result is that a conical shock wave is generated to equalise the difference of pressure. Therefore the useful flow region is restricted to this envelope and this causes a limitation the size of the model used in the test section. To reduce this blockage effect, the pressure surrounding the working section needs to be reduced.

\subsection{Comparisons with CFD (Figs 27-30)}

A numerical simulation of the intake entrance has been provided by Professor Hillier from Imperial College London. This was made for the actual flight Mach number of 6 , a condition which we can not duplicate. Consequently the test conditions are not identical but the main flow features should be similar.

These figures present two configurations: the first with the cowl lip placed upstream of the wedge shock (Figs 27 and 28) and the second with the cowl lip downstream (Figs 29 and 30). The correlation between CFD pictures and Schlieren photographs is clear and several similar flow features can be identified.

In Figs 27 and 28 the laminar separation behind the step and the reattachment shock are clearly visible. The extent of separation and the flow behaviour near the lower duct wall are comparable. The simulation also predicts the laminar separation on the inner cowl surface and a little downstream a pale region appears to be a reflected shock. In Figs 29 and 30 , the larger separated region behind the step is visible in both figures and there does not appear to be any separation on the inner cowl surface.

Comparison of the Schlieren pictures with the numerical predictions reveals overall agreement. The shock-wave pattern, separation and boundary layer thicknesses in the Schlieren pictures are also present in the simulation. Thus the CFD analysis supports the interpretation of the experimental data. A full description of the computational method and the results obtained is given in Refs 8 and 9 .

\subsection{Striations with the vortex generators present}

Schlieren pictures of the intake with vortex generators revealed some unusual effects. As shown in Fig. 32, striations or streamlines can be seen between the wedge shock and the ramp surface along the entire compression ramp. In addition, it should be noted that the path of these lines follows the profile of the body surface.

These streamlines might be explained by the formation of a wave system from the tip of the vortex generators. This wave system propagates downstream and reflects between the body surface and the wedge shock. Figure 31 presents a schematic diagram of this phenomenon which shows waves propagating along the ramp surface.

The waves reflect from the wedge shock, generating entropy surfaces which are sufficiently strong to be shown up on the Schlieren pictures.

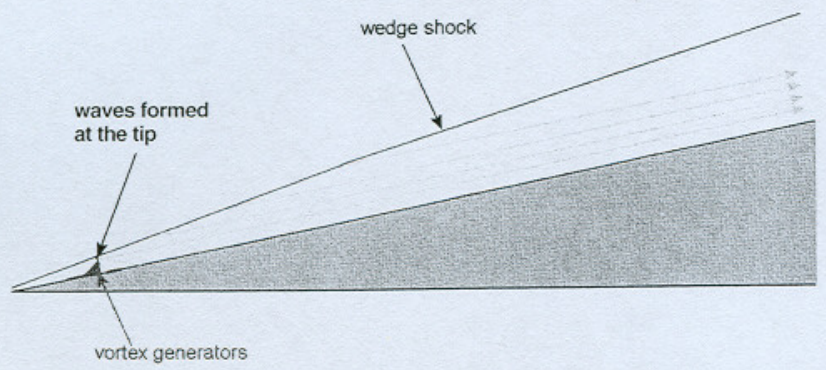

Figure 31. Waves system formed at the tip of the vortex generator and propagating out from the surface.

\subsection{CONCLUSIONS}

Experiments have been conducted to assess the intake flow starting process and to investigate the effect of various parameters on this process and on the flow structure. The effects of cowl position, Reynolds number, tripping the boundary layer and introducing blockage in the intake duct have been investigated and it was found that:

- Raising the cowl brings waves inside and increases the length of the separated region on the inner surface of the cowl, but reduces separation on the bottom surface of the intake.

- Reducing Reynolds number thickens the boundary layer and increases the boundary layer displacement thickness but has little effect on the intake flow.

- The vortex generators were too large and introduced a wave system which reflected from the leading edge shock wave.

- Filling in the step on the lower wall seems to have a minor effect on the flow pattern if the boundary layer is turbulent.

- Introducing a $1 \mathrm{~mm}$ wire has no effect on the intake flow.

- Introducing a $2 \mathrm{~mm}$ wire causes a blockage effect and prevents the intake from starting.

\section{ACKNOWLEDGEMENTS}

The authors are indebted to Professor Hillier from Imperial College, London, for supplying the two CFD pictures (Figs 27 and 29) and for his suggestion about the vortex generators. They also wish to thank Dr Terry Cain for his helpful assistance.

\section{REFERENCES}

1. CaIN, T. and WALTON, C. The sustained hypersonic flight experiment, AIAA Paper 2003-7030, 12th AIAA International Space Planes and Hypersonic Systems and Technologies, Norfolk Virginia, USA 15-19 December 2003.

2.. NeedHAM, D.A. Laminar Separation in Hypersonic Flow. PhD Thesis, University of London, 1965.

3. Nefdham, D.A. Progress report of the Imperial London College hypersonic gun tunnel. Imperial College of Science and Technology, Aero-TN 118, 1963.

4. Perigo, D. Shock Waves-Boundary Layer Interactions in a 2D Hypersonic Inlets. MSc Thesis, Cranfield University, College of Aeronautics, 1996.

5. Vannahme, M. Roughness Effects on Flap Control Effectiveness at Hypersonic speeds. MSc Thesis, Cranfield University, College of Aeronautics, 1994.

6. PrinCE, S.A. Hypersonic Turbulent Interaction Phenomena and Control Flap Effectiveness. MSc Thesis, Cranfield University, College of Aeronautics, 1995

7. Stollery, J.L. Hypersonic viscous interaction on curved surfaces, Ohio: Wright-Patterson Air Force Base, 1970. J Fluid Mechanics, 1970, 43, (3), pp 497-51.

8. BachChan, N. and Hillier R. Hypersonic Inlet Flow Analysis at OffDesign Conditions. AIAA Paper 2004-5380, 2004.

9. BACHCHAN, N and HILLIER R. Effects of hypersonic inlet flows non-uniformities on stablising isolator shock systems. AIAA Paper 2004 - 4716, 2004.

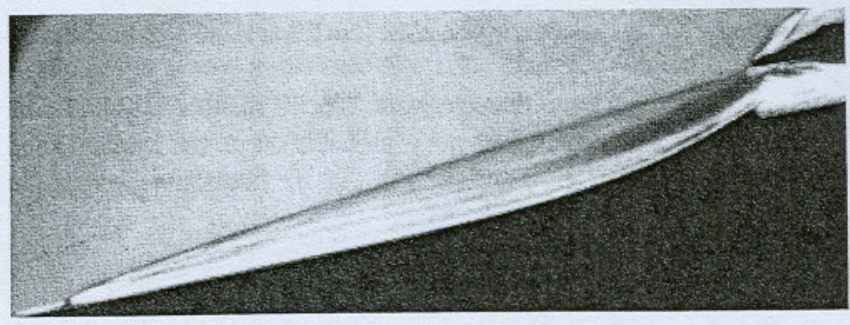

Figure 32. Illusration of a special flow feature associated with vortex generators. $7 \cdot 8 \mathrm{~mm} \operatorname{Re}_{n}=45,400 / \mathrm{cm}$ turbulent case 